\title{
Structural, Electronic and Mechanical Properties of Few-Layer GaN Nanosheet: A First-Principle Study
}

\author{
Vu Ngoc Tuoc ${ }^{1, *}$, Le Thi Hong Lien ${ }^{1, *}$, Tran Doan Huan ${ }^{2}$ and Nguyen Ngoc Trung ${ }^{1}$ \\ ${ }^{1}$ Institute of Engineering Physics, Hanoi University of Science and Technology, 01 Dai Co Viet Rd., Hai Ba Trung Dist., Hanoi 100000, \\ Vietnam \\ ${ }^{2}$ School of Materials Science and Engineering, Georgia Institute of Technology, 771 Ferst Drive NW, Atlanta, Georgia 30332, United States
}

\begin{abstract}
The low-dimensional III-V semiconductors are interesting candidate materials for the tailoring of two dimensional (2D) layered structures. We have performed the first-principles calculations on the structural, electronic, and mechanical properties of few-layer gallium nitride (GaN) nanosheet, formed from various bulk phases and stacking patterns, to investigate the effects of structural modification and sheet thickness on their structural, electronic, and mechanical properties. We observed that with the thickness increases, few-layer GaN nanosheets have suffered from the size-induced transition from indirect semiconductor to metallic as well as from the graphitic - planar honeycomb to the wurtzite buckled 2D form. Optimized geometries, binding energy, phonon spectra, electronic band structure, and elastic tensor calculation has ensured the dynamical and mechanical stability of the sheet. Our study indicates that there are two competing mechanisms that govern the polarity compensation with the sheet's net dipole served as an important parameter for determining the sheet's stable formation.

[doi:10.2320/matertrans.MT-MN2019030]
\end{abstract}

(Received January 9, 2020; Accepted March 16, 2020; Published May 1, 2020)

Keywords: DFT, nanosheet, polarity compensation, ultrathin

\section{Introduction}

Since the experimental discovery of freestanding singlelayer graphene ${ }^{1)}$ having extraordinary physical properties as a two-dimensional (2D) material, scientific interest has concentrated on the emergence of graphene-like 2D layered structures. A series of this new class of materials whose properties differ from those of their three-dimensional (3D) and one-dimensional (1D) counterparts have successfully synthesized 2D, including $\mathrm{SiC},{ }^{2)} \mathrm{BN}^{3}, \mathrm{MoS}_{2},{ }^{4)} \mathrm{ZnO},{ }^{5}$ $\mathrm{SnS}_{2},{ }^{6)}$ and $\mathrm{WSe}_{2}{ }^{7)}$ Later predicted $2 \mathrm{D}$ III-V materials ${ }^{8,9)}$ are the new addition to this class of 2D nanosheet (NS) crystal. The search for $2 \mathrm{D}$ materials exfoliated from parent compounds now is extensively implemented within recently emerged high-throughput van der Waals (vdW) density functional theory (DFT) simulation suggesting a series of compounds potentially to engineer novel properties by stacking different $2 \mathrm{D}$ materials in a desired fashion. ${ }^{10-12)}$

To date suggesting novel $2 \mathrm{D}$ materials with unique electronic and optical properties of low-dimensional systems are in the focus to create vdW hetero-junctions regarding next-generation nanodevices in electronic and optoelectronic technology. Recently technological efforts in field-effect transistors saw a shift to the channel made from multiple separated NSs encapsulated by the gate referred to as "gateall-around $3 \mathrm{~nm}$ technology" showing potential for continued device scaling. ${ }^{13)}$

Therefore, research on group-III nitride semiconductors 2D materials, and $\mathrm{GaN}$ in particular, that has been performed in ultra-thin films of few nanometers in thickness, expected to open up an avenue in applicable optoelectronic devices. ${ }^{14)}$ Recently, A. Balushi et al. ${ }^{15)}$ have succeeded in obtaining a graphitic 2D mono-layer GaN sheet using the migration-

*Corresponding authors, E-mail: tuoc.vungoc@hust.edu.vn, lien. lethihong@hust.edu.vn enhanced encapsulated growth. Additionally, theoretical prediction within $\mathrm{DFT}^{8,9,13,16-20)}$ has been proposed that GaN nanosheet (NS) transforms into a 2D graphitic structure while forming the ultra-thin film. Therein, it is reported that a graphitic GaN monolayer (ML) has an indirect gap $(\sim 2 \mathrm{eV})$ and its electronic structure is very sensitive to its thickness, to an external electric field ${ }^{16)}$ and applying strain. ${ }^{18,20)}$ Further studies $^{21)}$ show that the nanoscale phonon transport in ML $\mathrm{GaN}$ within the framework of BTE showing orbitally driven low thermal conductivity $\kappa$ of ML GaN and it's prospective for applications in thermoelectric.

In the graphitic phase, the cations and anions are arranged in trigonal-planar coordination, so that the two polar surfaces now merges into one honeycomb layer. This removes the intrinsic surface dipoles and thus stabilize the sheet. This is a favored mechanism for the stability of the 2D polar ultrathin sheets originated from the wurtzite (WZ) structure. However, this stability is reported to limit to a thickness of only a few atomic layers (up to five honeycomb layers ${ }^{8,9,22)}$ ). Another mechanism of surface polarity compensation is the charge transferring and metallization of the outer polar surface - which is favorable with a thicker sheet. In this work, we examine the few-layer - up to eight graphitic honeycomb layers and eight bi-layer of polar surfaces form 2D GaN NS's structure. Our detail Bader charge and dipole moment analysis shows that these are the two competing mechanisms of stability governed the transformation from the exotic graphitic form to the WZ materials, with extension of the NS thickness. Moreover, these 2D structures also exhibit novel properties different from their bulk counterparts such as the transition from direct to indirect band gap, and modification to the electronic structure depending on stacking patterns between layers without having dangling bonds. However different from the previous studies, we have included in study all the three possible stacking patterns $\mathrm{A}, \mathrm{AB}, \mathrm{ABC}$, of the honeycomb stacking and indicate that 


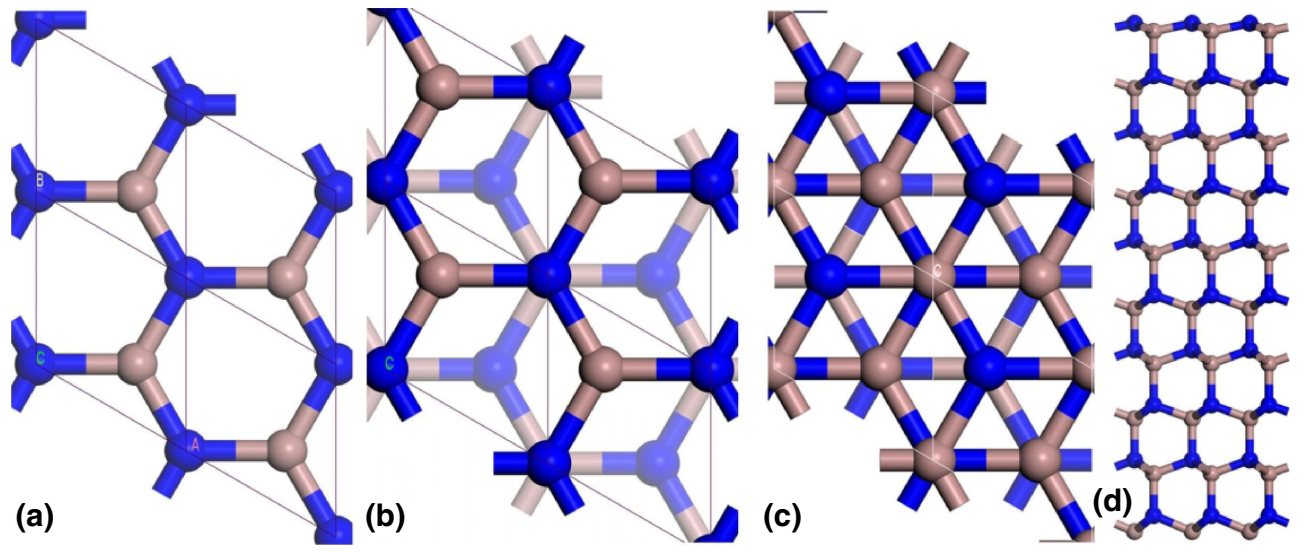

Fig. 1 Image of the simulated NS, in-plane view of the structures (a) g-GaN-A-1, (b) g-GaN-AB-3, (c) g-GaN-ABC-3 and cross-view of (d) w-GaN-6, the blue and gray balls represent $\mathrm{Ga}$ and $\mathrm{N}$ atoms respectively, and the thin line represents the unit cell and blur is complementary cues to depth.

the newly introduced stacking sequence, i.e. $\mathrm{ABC}$, are the most dynamically and mechanically stable for the NS with thickness range up to only four graphitic layer.

\section{Computational Methods}

The calculations in this work are based on DFT using the projector-augmented wave (PAW) method as implemented in the plane-wave code VASP DFT. ${ }^{23-25)}$ For the structural relaxations and energy calculations, we employ the generalized gradient approximation (GGA) with the Perdew-Burke-Ernzerhof $(\mathrm{PBE})^{26)}$ parametrization for most calculations unless mentioned otherwise. An energy cutoff of $600 \mathrm{eV}$ for the plane-wave basis set is used throughout all calculations and ensures accuracy of the energy of $10^{-4}$ $\mathrm{eV} /$ atom and elastic constants of about $1 \mathrm{~N} / \mathrm{m}$, for the $2 \mathrm{D}$ material. The k-point sampling uses the Monkhorst-Pack ${ }^{27)}$ scheme and employs a $12 \times 12 \times 1$ mesh for the PBE functional. The vdW interactions corrections, which is related to polarizability, between the $2 \mathrm{D}$ materials are modeled using the DFT-D3 method of Grimme. ${ }^{28)}$ For the 2D structures, a vacuum spacing of $12 \AA$ ensures that the interactions between the layer's images are negligible. Example structures are shown in Fig. 1.

\section{Results and Discussions}

\subsection{Structural stability}

To identify the suitable candidate configurations for our 2D materials we have started from all known 3D crystal phases. For GaN materials, the relevant 3D crystal phases are $\mathrm{WZ}$ and zinc blende (ZB). So that, our few-layer NS has been created with three stacking patterns, i.e. $\mathrm{A}, \mathrm{AB}$ and $\mathrm{ABC}$, by (i) taking multiple of the honeycomb ML prototype of graphene, (ii) similar already created in Refs. 8, 9, 22) cutting from the frozen crystal structure of $\mathrm{WZ}$ with a perpendicular surface to the c axis to create a (001) thin film and (iii) our newly introduced in the ZB phase with (111) surface respectively. Thus the forming NS has that is also known as $\mathrm{A}, \mathrm{AB}$ and $\mathrm{ABC}$ stacking sequences (see Fig. 1). The two later NS types always lead to a cation-terminated surface at one side and an anion-terminated surface at the other side, that is the so-called Tasker type 3 polar surfaces, ${ }^{29,30)}$ which are intrinsically unstable due to the divergence of the dipole moment and the surface energy. After performing fully geometry relaxation for all NSs, the optimized structure geometries show that the two kinds of the structure's reconstruction have occurred. First, for the thinner NS from WZ frozen phase, i.e. the AB stacking pattern, with less than ten polar surfaces, the NSs are no longer in the buckled form with two closed different polar surfaces but becoming perfectly flat honeycomb, i.e. hexagonal, structure with no extra bonds along the [001] similar to as was previously reported for wurtzite $\mathrm{GaN}$ and other III-V compounds thin NSs. ${ }^{22,30)}$ In addition, we found that our newly supposed fewlayer NSs - cutting from the frozen ZB phase, i.e. the ABC stacking pattern, have also adopted a graphitic honeycomb flat structure. And the second, while going beyond ten polar surfaces, it was found that after structure relaxation, our structures still preserve the parent form with the outermost polar surfaces are shrunk and $\mathrm{N}$-face goes more inside due to the effect of reconstruction. Since our few-layer NSs are always defined with number of bi-layer, i.e. Ga-face and Nface, ensuring equal composition content, our NS's structure are labeled as follows: NS that contains the merged two polar surface layer into one graphitic honeycomb layer, from here it is named as a layer, are denoted as "g-GaN". So that, the "g-GaN-AB-3" and "g-GaN-ABC-3" are the NS with three graphitic layers with stacking sequences $\mathrm{ABAB}$ and $\mathrm{ABCABC}$, originating from $\mathrm{WZ}$ and $\mathrm{ZB}$ phases, respectively. While still buckled bi-layer NSs from the WZ phase are denoted as w-GaN, e.g. w-GaN-6 is WZ NS with six buckled bi-layer. Our structural relaxations are performed for $\mathrm{GaN}$ NSs with numbers of bi-layers ranging up to eight. We have performed fully optimization of all structures until the forces and in-plane stresses are converged to within $1 \mathrm{meV} / \AA$ and $0.01 \mathrm{GPa}$, respectively.

The values of the structural parameters for the most stable structures studied in this work are given in Table 1. The lattice constants of the optimized bulk WZ GaN ( $\left.\mathrm{P}_{3} \mathrm{mc}\right)$ are $\mathrm{a}=\mathrm{b}=3.21 \AA, \mathrm{c}=5.22 \AA$ and $\mathrm{ZB}(\mathrm{F}-43)$ is $4.52 \AA$ which is consistent with the previous DFT studies. ${ }^{31)}$ Average Ga-N bond lengths are the same in two phases $(1.96 \AA)$ and bond angles are $109.47^{\circ}$, which evident the $s p^{3}$ hybridization. After 
Table 1 Structural characteristic table of the selected NSs.

\begin{tabular}{|l|c|c|c|c|c|c|c|c|}
\hline \multicolumn{1}{|c|}{ Structure } & $\begin{array}{c}\mathrm{E}_{\mathrm{Fo}} / \text { At } \\
(\mathrm{eV})\end{array}$ & $\begin{array}{c}\mathrm{E}_{\mathrm{Col}} / \text { At } \\
(\mathrm{eV})\end{array}$ & $\begin{array}{c}\text { In-plane } \\
\text { lattice } \\
(\AA)\end{array}$ & $\begin{array}{c}\text { Avr. Ga-N } \\
\text { bond length } \\
(\AA)\end{array}$ & $\begin{array}{c}\text { Avr.Ga- } \\
\text { Nbond } \\
\text { angle }(\mathrm{o})\end{array}$ & $\begin{array}{c}\text { Distance } \\
\mathrm{b} / \text { w layer } \\
(\AA)\end{array}$ & $\begin{array}{c}\mathrm{C}_{11} \\
(\mathrm{~N} / \mathrm{m})\end{array}$ & $\begin{array}{c}\mathrm{C}_{12} \\
(\mathrm{~N} / \mathrm{m})\end{array}$ \\
\hline g-GaN-1 & 0.482 & -1.597 & 3.206 & 1.851 & 120.0 & - & 138.511 & 61.198 \\
\hline g-GaN-AB-2 & 0.433 & -1.646 & 3.275 & 1.853 & 120.0 & 3.196 & 257.056 & 124.521 \\
\hline g-GaN-ABC-3 & 0.413 & -1.666 & 3.285 & 1.854 & 120.0 & 3.149 & 369.581 & 181.261 \\
\hline GaN-ABC-4 & 0.401 & -1.678 & 3.289 & 1.856 & 120.0 & 3.08 & 489.322 & 240.849 \\
\hline g-GaN-ABC-5 & 0.394 & -1.685 & 3.301 & 1.857 & 119.9 & 3.06 & -94.822 & 564.001 \\
\hline w-GaN-6 & 0.289 & -1.789 & 3.239 & 1.936 & 109.44 & - & - & - \\
\hline w-GaN-7 & 0.250 & -1.829 & 3.227 & 1.949 & 109.42 & - & - & - \\
\hline w-GaN-8 & 0.219 & -1.859 & 3.218 & 1.943 & 109.44 & - & - & - \\
\hline
\end{tabular}

relaxation, optimized graphitic structures ( $\mathrm{g}-\mathrm{GaN}$ series) have slightly larger in-plane lattice constant raising from $3.206 \AA$ to $3.22 \AA$ together with the $\mathrm{Ga}-\mathrm{N}$ bond length is in the range $1.85 \AA-1.86 \AA$ while going from g-GaN-1 to g-GaN-5, and all with the bond angle $120^{\circ}$, i.e. a sign of the $s p^{2}$ hybridization, essentially different from that of WZ bulk phase, (see Table 1). At the same time, the distance between layers have shortened from $3.20 \AA$ to $3.08 \AA$ going from $\mathrm{g}-\mathrm{GaN}-2$ to g-GaN-5, which are the evidence of no covalent bonding between the layers and like graphite, in these structures, the atoms $\mathrm{Ga}$ and $\mathrm{N}$ are covalently bonded to each other in-plane and the interaction between the layers in the vertical direction to this plane is the weak vdW attraction forces. This attraction causes shortening the interlayer distance and very slightly widening the in-plane lattice. There are almost no differences with different stacking pattern in bond's statistics. In the buckled structure, (from the 6 layer up) there is also very slightly widening the in-plane lattice (Table 1) but that is due to the reconstruction of the surface-layer with shortening $\mathrm{Ga}-\mathrm{N}$ bond in $\mathrm{N}$-face to $1.90 \AA$, causing the $\mathrm{N}$-face to go in-side the NS (see the Fig. 1(d)) while almost non-changed in the Ga-face $(1.95 \AA)$.

For the energetic stability, the cohesive energy - the difference between the average energy of the free atoms and that of the atoms in a condensed state, are also given in Table 1. It is determined in term of per-atom energy by means of $E_{\mathrm{Coh}}=\left(E_{\mathrm{NS}}-n E(\mathrm{Ga})-n E(\mathrm{~N})\right) / 2 n$, where $E_{\mathrm{NS}}$ is the total energy of GaN NS, $E(\mathrm{Ga})$ and $E(\mathrm{~N})$ are the total energies of isolated atoms, $n$ represents the number of $\mathrm{Ga}$ or $\mathrm{N}$ atoms in the NS. Noted that only data for stable representative NS are listed. The value of cohesive energy in a range of $1-2 \mathrm{eV} /$ atom show that the NS is in the semiconductor state. Furthermore, we also estimate the formation energy of proposed few-layer NS with respect to their most stable bulk form (WZ), which is determined by the energy difference $E_{\mathrm{Form}}=E_{\mathrm{NS}} / N_{\mathrm{NS}}-E_{3 \mathrm{D}} / N_{3 \mathrm{D}}$, where $E_{\mathrm{NS}}$ and $E_{3 \mathrm{D}}$ are the energy of the GaN NS and 3D bulk (WZ) system, respectively, $N_{\mathrm{NS}}$ and $N_{3 \mathrm{D}}$ refer to the number of atoms in the corresponding unit cells.

The NS's formation energies relative to WZ phase of GaN range from $0.39-0.48 \mathrm{eV} /$ atom for g-GaN-ABC series, while $0.22-0.29 \mathrm{eV} /$ atom for $\mathrm{w}-\mathrm{GaN}-6$ to $\mathrm{w}-\mathrm{GaN}-8$ structures. These values are comparable to that of GaN ML, which has been predicted ${ }^{8,9,30)}$ and that of $\mathrm{SiC}$ ML which has been reported to fabricate successfully. ${ }^{2)}$ This relatively high formation energy w.r.t. stable bulk phase have suggested that it might be feasible to deposit GaN NSs on suitable substrates, e.g. with hexagonal symmetry, in order to reduce the formation energies and stabilize the structures.

It can be followed from Table 1 that, for cohesive energy, a minus sign shows the energy gained by arranging the atoms in an NS state. By increasing the number of layers, the lowering of energy is originated from the stacking effect and indicates the stability of forming multiple-layer NS. At the same time, when going beyond the six bi-layers, the buckled WZ NS has higher cohesive binding energy compare to that of the ZB phase, showing that the more energetically stable it is. There is almost no difference in biding energy related to different stacking patterns between graphitic layers.

To investigate the thermodynamical stability of all structures, phonon spectra of the NSs are calculated by using the VASP connected with Phonopy code. ${ }^{32)}$ The phonon spectra of several representative NS structures shown in Fig. 2, varying in the number of layers, stacking patterns and reconstructions, i.e. g-type or w-type. From 2(a) to 2(e) is phonon dispersion curves for five graphitic phases, i.e. g-GaN-1, g-GaN-AB-2, g-GaN-ABC-3, -4, -5 which show no imaginary for all calculated frequencies in the Brillouin zone, demonstrating that they are all dynamically stable. Noted that the phonon at high-symmetry $\mathrm{k}$ points of our mono-layer agrees with previous studies. ${ }^{8}{ }^{8}$ It is also worth noting that from the three to five graphitic layer the thermodynamically stable structures are the NS with $\mathrm{ABC}$ stacking sequences, which are originated from ZB (111) cuttings, however not that one with $\mathrm{AB}$ stacking, originating from WZ (001) cuttings, as proposed in Ref. 22). For instance, in Fig. 2(f) and Fig. 2(g), we found the phonon spectra with imaginary frequencies for g-GaN-AB-3 around $\mathrm{K}$ symmetry point, i.e. $\{1 / 3,1 / 3,0$.$\} , and for g-GaN-AB-4$ around $\mathrm{K}$ and $\mathrm{M}$, i.e. $\{1 / 2,0.0$.$\} . Further, the phonon spectra$ of our buckled w-GaN series from six to eight bi-layer also contain imaginary curves (see Fig. 2(h) and 2(i)), arguing that they are not dynamically stable although are energetically favorable. A possible reason for this instability is the dipole moment in the direction perpendicular to the NS surface (see discussion in Section 3.3). And thus, only for much thicker $\mathrm{w}-\mathrm{GaN}$, the reconstruction, need to that reduce the dipole moment, could stabilize the structure. The other 

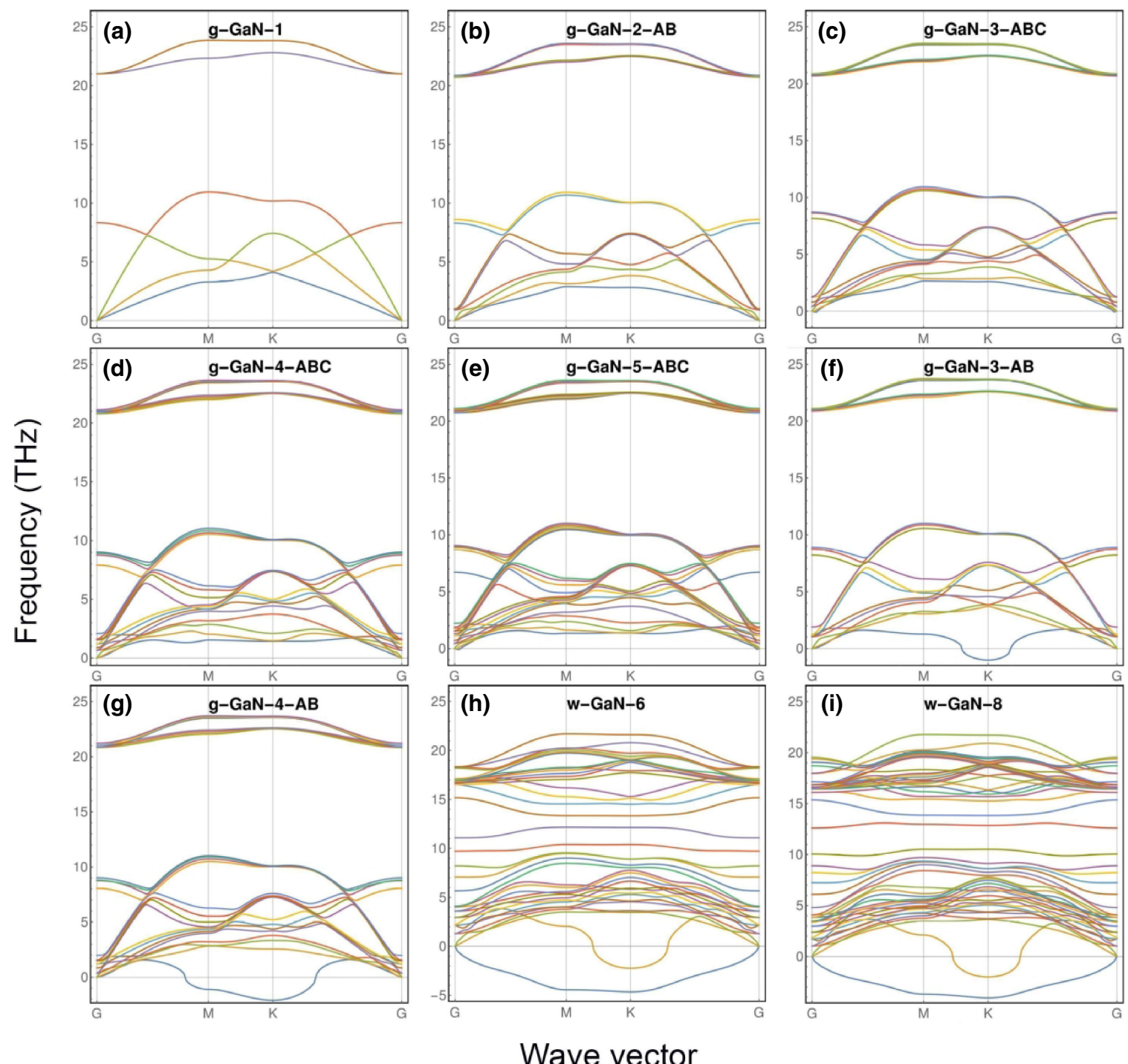

Wave vector

Fig. 2 Phonon spectra of selected NS, with the different numbers of layers, stacking sequence and reconstruction kind.

stacking pattern as of AA is also not given here due to its unstable phonon spectra containing imaginary frequencies.

Our phonon calculation is the test of whether a proposed 2D configuration would be dynamically stable, or the structure represents a minimum of the potential energy surface. It argues that there is are only five dynamical stable graphitic NS's configurations with up to five-layer. Additionally, we have also performed a test for the distortion of the shape of NS's unit cell, by calculating the components of the stiffness tensor corresponding to uniaxial deformations along the major axis, i.e. the $C_{11}, C_{22}$ and $C_{12}$ components in the Voigt notation. As suggested in Refs. 10,11) the material structure is considered as mechanically stable if the two following criteria are met (i) neither $C_{11}$ and $C_{22}$ is negative and (ii) $C_{11}>\left|C_{12}\right|$. Our elastic calculation is based on the vaspkit utility ${ }^{33)}$ and is given in Table 1 as component $C_{11}$ and $C_{12}$ (in our hexagonal NS's symmetry the $C_{11}=C_{22}$ ). The results show that the g-GaN-ABC-5 has failed to meet both the above criteria as $C_{11}$ is negative and $C_{11}>\left|C_{12}\right|$. This suggested that there are left only four mechanically stable graphitic NS configurations with up to four layers.

\subsection{Electronics structure}

Following the NS stability studies, we come to the study on the NS electronic structure and the effect of the reduced dimensionality on their electronic structures. Bulk 3D GaN semiconductors with a direct band gap of $3.2 \mathrm{eV}$ and its band gap can be tunable in the wide range of visible and ultraviolet (UV) when forming low-dimensional structure and/or heterojunction with other group-III nitrides like AlN. ${ }^{34)}$ Our DFT GGA band gap of bulk GaN is $1.69 \mathrm{eV}$, which is consistent with previous GGA calculations. ${ }^{31)}$ Noted that it is still much smaller than the experimental value $(3.2 \mathrm{eV})$. Although the recent hybrid functional calculation, e.g. HSE0 $6^{31)}$ represent most accurate to evaluate the bandgap, they are very time consuming and prohibitively expensive as the structure's size grows, what is in our case. The choice of DFT-GGA based calculations could be enough in this work to study the band structure and the trend of gap changes in thin GaN NSs. The values of the electronics parameters for the most stable structures studied in this work are given in Table 2. All the band structures of the thin graphitic NS show similar shapes with the common feature as an indirect semiconductor gap - the valence band maximum (VBM) is 
Table 2 Electronic characteristic table of the selected NSs.

\begin{tabular}{|l|c|c|c|c|}
\hline Structure & $\begin{array}{c}\text { Band gap } \\
(\mathrm{eV})\end{array}$ & $\begin{array}{c}\text { NS Work } \\
\text { function }(\mathrm{eV})\end{array}$ & $\begin{array}{c}\text { Electron effective mass } \\
\left(m_{\mathrm{e}}\right)(\mathrm{at} \mathrm{G-K,G-M})\end{array}$ & $\begin{array}{c}\text { Hole effective mass } \\
\left(m_{\mathrm{h}}\right)(\mathrm{at} \mathrm{K}-\mathrm{G}, / \mathrm{K}-\mathrm{M})\end{array}$ \\
\hline g-GaN-1 & 2.18 & 5.08 & $0.254 / 0.254$ & $-1.116 /-1.143$ \\
\hline g-GaN-AB-2 & 1.88 & 5.14 & $0.226 / 0.226$ & $-2.703 /-2.497$ \\
\hline g-GaN-ABC-3 & 1.18 & 5.16 & $0.220 / 0.220$ & $-2.132 /-2 / 314$ \\
\hline GaN-ABC-4 & 0.77 & 5.04 & $0.223 / 0.223$ & $-2.214 /-2.401$ \\
\hline g-GaN-ABC-5 & 0.16 & 5.12 & $0.229 / 0.229$ & $-2.245 /-2.434$ \\
\hline w-GaN-6 & metallic & 5.91 & - & - \\
\hline w-GaN-7 & metallic & 6.00 & - & - \\
\hline w-GaN-8 & metallic & 5.96 & - & - \\
\hline
\end{tabular}
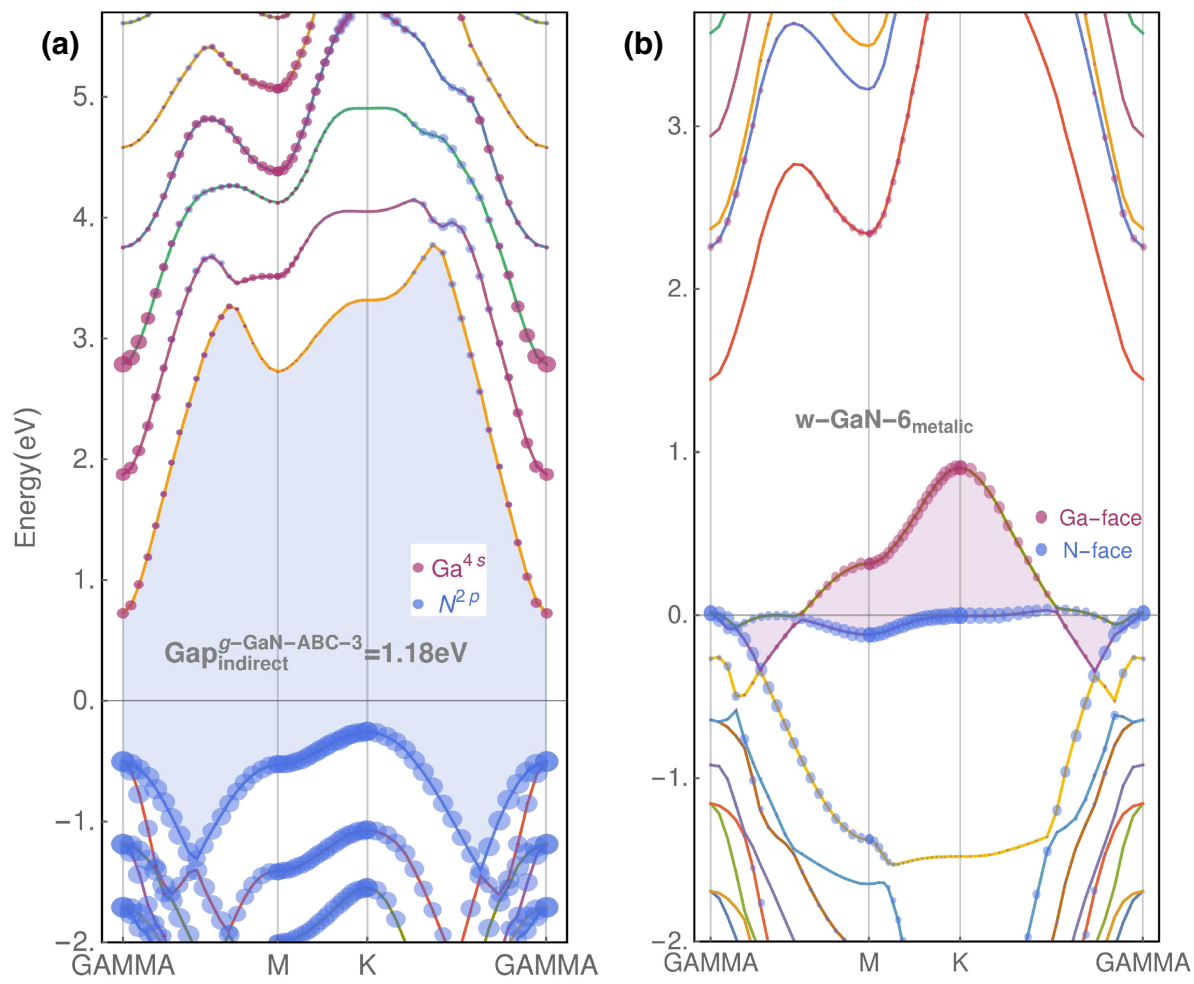

Fig. 3 Band structure of the g-GaN-ABC-3 (left) and w-GaN-6 with projected on the Ga4s and N2p orbitals (a) and the polar surface states (b).

located at the $\mathrm{K}$ symmetry point whereas the conduction band minimum $(\mathrm{CBM})$ at the Gamm $(\{0.0 .0\}$.$) . In other words,$ the major difference from the corresponding bulk 3D band of $\mathrm{GaN}$ is direct to indirect gap transitions for all 2D thin GaN film. This is the results from the electronic structure depending on stacking patterns without having dangling bonds. In other word this transition in the bandgap character due to dimension reduction and changing from $s p^{3}$ to $s p^{2}$ hybridization (see Fig. 3(a)). Further, their band gap decreases smoothly with the increasing thickness (see Table 2) serving a way to tune the gap of the semiconductor NSs, that is within the range of visible light, suitable for optoelectronic and photocatalytic applications.

In contrast, the buckled WZ NSs are in the metallic states due to the intrinsic surface states arising from the polar surface's unsaturated bonds. Figure 3(b) shows the projected band structure on the outer polar surface, forming states at the Fermi level. In 2D NSs system, i.e. with high intrinsic surface-to-volume ratio compared to bulk materials, surface reconstructions and surface states play a dominant role in determining its properties. This is the effect of metallization as a stabilization mechanism for polar surfaces (see Section 3.3).

The next important parameter for semiconductor structures is the effective mass of electron and hole defining their carrier mobility. In Table 2 the effective masses $m^{*}{ }_{\mathrm{e}} / m^{*}{ }_{\mathrm{h}}$ of the graphitic GaN NS are given. Their electron effective mass is comparable to that of the silicon $\left(m^{*}{ }_{\mathrm{e}}=0.26 m_{\mathrm{e}}\right)$ and smaller than that of single-layer $\operatorname{MoS}_{2}\left(0.483 m_{\mathrm{e}}{ }^{8)}\right)$ which is the emerging materials for future electronics devices. This also convinces the potential of application proposed 2D III-V NS materials for electronics applications.

Since GaN is a strong piezoelectric material, the local (bilayer) polarization and its field are investigated in the proposed NS by plotting the planar and macroscopicaveraged $^{31)}$ electrostatic potential. The averaged potentials of the two representatives of the proposed NS are shown in Fig. 4. The planar average of the potential is obtained by 

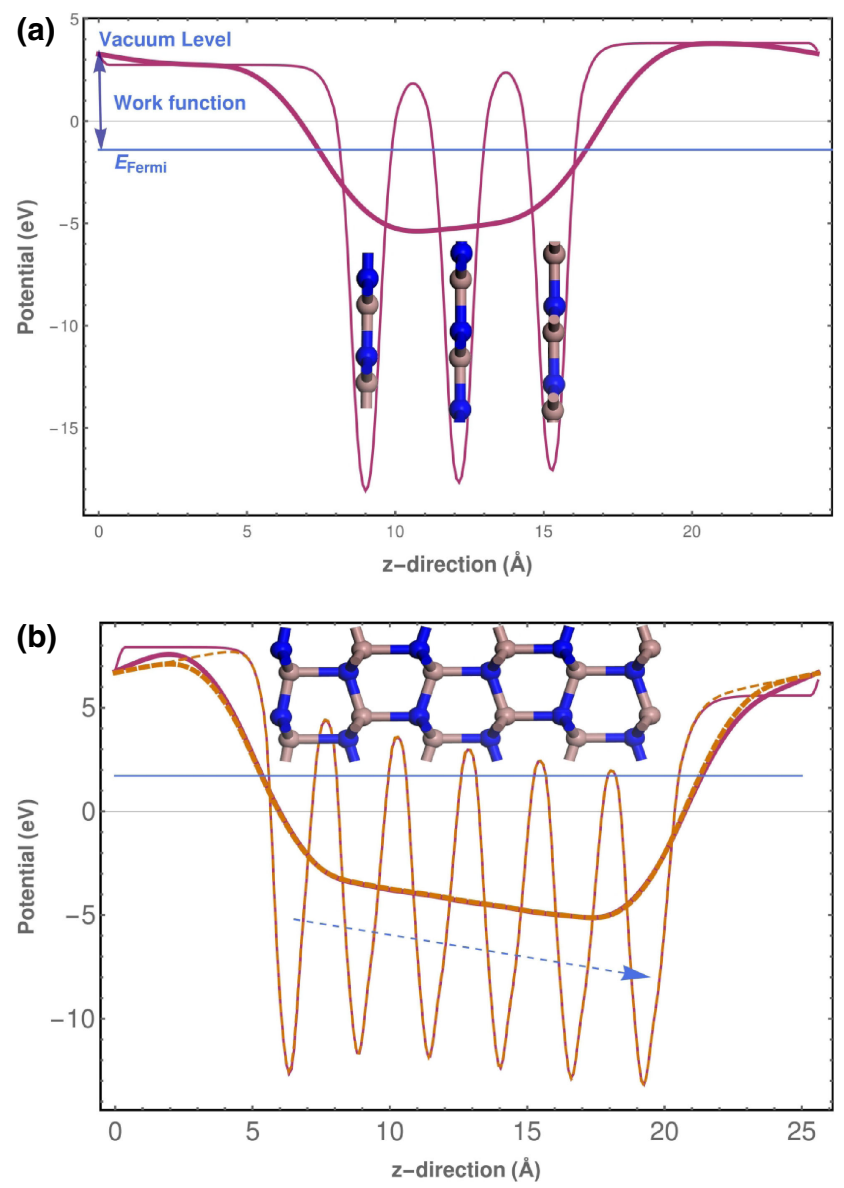

Fig. 4 Planar (thin line) and macroscopic (thick line) averaged electrostatic potential of g-GaN-ABC-3 (a) and w-GaN-6 (b) with (solid line) and without (dashed line) dipole correction.

averaging potential values within a plane perpendicular to the surface of the NS. The macroscopic-averaged potential is obtained by taking averages of the planar average potential over distances of the repeated unit of the NS along the perpendicular direction. With the given averaged electrostatic potential one can define the work function of the NS as the difference between the vacuum level and the Fermi energy (illustration is given in Fig. 4(a)) and listed in Table 2. The work function of the NSs is increasing with the NS thicknesses. To correct the effects of imposing periodic boundary conditions we have used the dipole corrections to the total energy and the Hellman-Feynman forces. ${ }^{35-37)}$ In Fig. 4(b) the dipole corrected potential lines (the solid line in red) have been shown together with the without-dipolecorrected lines (the dashed line in yellow) to check their differences. This correction introduces a jump in the potential $\mathrm{V}(\mathrm{r})$, which is placed in the middle of the vacuum region and cancels the artificial field in the vacuum region. An estimate of the macroscopic internal field inside the NS can be obtained from their slope and the thin blue dashed arrow in the Fig. 4(b) shows the direction of the internal dipole moment of the polar surfaces.

\subsection{Competing mechanism for polarity compensation}

Figure 4 shows the difference averaged electrostatic potential picture between the graphitic $(\mathrm{g}-\mathrm{GaN})$ and $\mathrm{WZ}$ (w-GaN) NS, which also illustrates the role of the electro-

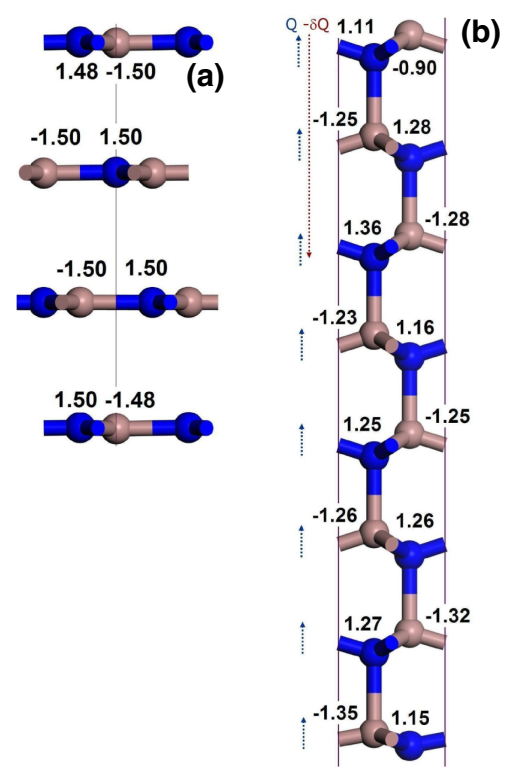

Fig. 5 Bader charge in relaxed (a) g-GaN-ABC-4 and (b) w-GaN-8.

static interaction in the stabilization of the planar and buckled geometries. This indicates that the alternating buckled bilayer structure of GaN NS results in a dipole moment across the NS. To estimate this dipole moment we have performed an analysis on Bader charge ${ }^{38)}$ - a net atomic charges, describing how much charge is transferred from Ga to N, which is connected to the electron negativity. The relatively large electron negativity difference of $\mathrm{Ga}$ and $\mathrm{N}$ atoms results in large ionic charges, in terms of Bader charges, reflected the asymmetric distribution of electrons in $\mathrm{Ga}-\mathrm{N}$ bonds. Through the Bader analysis, we found that the atomic charges are different in graphitic and WZ NS. In stable graphitic NS, charge transfer in $\mathrm{Ga}-\mathrm{N}$ bond is about $1.50|\mathrm{e}|$ and no charge transfer between the layers (see Fig. 5(a)). Whereas in the relaxed WZ NS the Bader charges are different with different layers, close to the bulk values $\left(Q_{\text {bulk }} \sim 1.27\right.$ e in WZ 3D phase) inside the NS and highly confined at the outer surfaces. The reason is involved in the mechanism that stabilizes the polar surface. It can be explain in the simple illustration (Fig. 5(b)) that the charge transfer at the polar surface can be considered as a difference of $Q$ and an excess charge $\delta Q$, which in turn forms an addition surface dipole (red long dashed arrow in Fig. 5(a)) in an opposite direction, compensating the internal polar surface dipole (blue dashed arrows).

In general, the polarity compensation has always occurred at the polar material surfaces/interfaces or nanostructures. This compensation could be achieved by a mechanism as modification electron charge density at the surface layers, i.e. surface reconstruction, resulting in the metallization of the surface layers. However for ultrathin as in a case of the few-layer or even ML NS, the charge cannot be enough reconstructed. Therefore the NS compensation can be realized by a novel mechanism involving a transition to a graphitic structure by merging the two polar surfaces and removing the dipole. This is the pre-critical regime with possible strong atomic relaxation. In Fig. 6 we proposed the plot of the dependence of the dipole moment vs. NS 


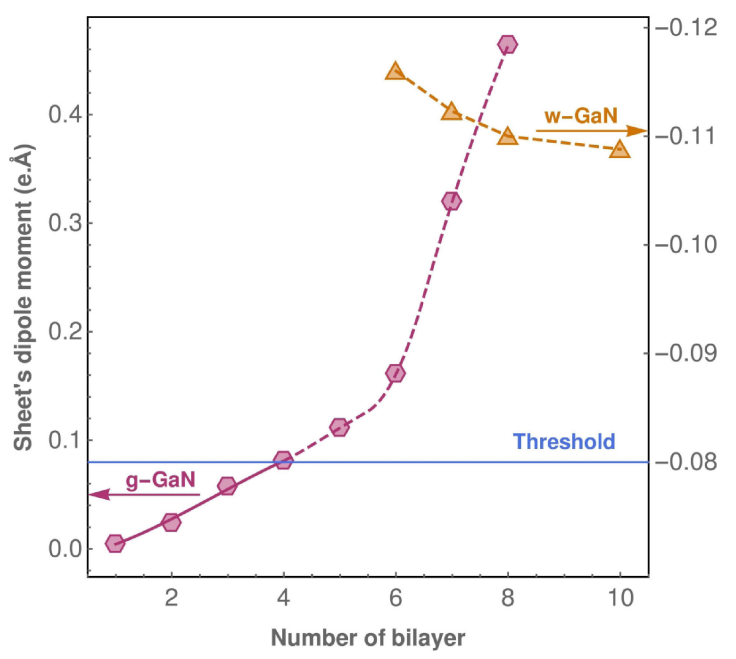

Fig. 6 NSs dipole moment depends on the number of the bi-layer.

thickness. The solid line denotes the stable NS configurations and the blue line marked the threshold dipole moment for the stable structure. This shows the competing of the two above mechanisms for polarity compensation depending on the NS's net dipole moment. The thinner sheet lowering the dipole by adopting a graphitic structure with $s p^{2}$ hybridization. These ultrathin NS are only thermodynamically and mechanically stable with up to four-layer (as in Section 3.1). Beyond it, the net dipole is quickly diverse up (red dashed line with hexagonal symbol). In contrast, the net dipole for the WZ NS (yellow dashed line with triangular symbol) stabilizing down with increasing the number of bi-layers by reconstructing the surface layer and driving the charge transfer from the anion- to the cation-surface. So that the thin blue line might play a role as a threshold for compensating net dipole moment to define the stable structure for the WZ NS.

\section{Conclusion}

Few-layer thin NS materials not only represent the scaling down in the thickness film, but might also bring a variety of novel electronic, optical, and mechanical properties for potential electronics and photo-catalytic device's applications. We have identified structures of the few-layer GaN with planar honeycomb and buckled hexagonal structure. Among the three types of stacking patterns, the ABC pattern are most stable and the graphitic NS with the indirect gap can be dynamically and mechanically stable up to only four layers. As the result of averaging electrostatic potential and the Bader analysis, there are two competing mechanisms that govern the polarity compensation in which the net dipole can serve as a threshold quantity determining the NS stable formation.

\section{Acknowledgments}

The authors are grateful for the financial support by the
Vietnam Ministry of Education and Training (MOET), under grant number B2018-BKA-57. The authors also acknowledge computational support from XSEDE under Grant No. TGDMR170031.

\section{REFERENCES}

1) K.S. Novoselov, A.K. Geim, S.V. Morozov, D. Jiang, Y. Zhang, S.V. Dubonos, I.V. Grigorieva and A.A. Firsov: Science 306 (2004) 666.

2) S.S. Lin: J. Phys. Chem. C 116 (2012) 3951.

3) C. Jin, F. Lin, K. Suenaga and S. Iijima: Phys. Rev. Lett. 102 (2009) 195505.

4) K.F. Mak, C. Lee, J. Hone, J. Shan and T.F. Heinz: Phys. Rev. Lett. 105 (2010) 136805.

5) C. Tusche, H.L. Meyerheim and J. Kirschner: Phys. Rev. Lett. 99 (2007) 026102.

6) H. Zhong, G. Yang, H. Song, Q. Liao, H. Cui, P. Shen and C.X. Wang: J. Phys. Chem. C 116 (2012) 9319.

7) H. Fang, S. Chuang, T.C. Chang, K. Takei, T. Takahashi and A. Javey: Nano Lett. 12 (2012) 3788.

8) H.L. Zhuang, A.K. Singh and R.G. Hennig: Phys. Rev. B 87 (2013) 165415.

9) A.K. Singh, H.L. Zhuang and R.G. Hennig: Phys. Rev. B 89 (2014) 245431.

10) S. Haastrup et al.: 2D Mater. 6 (2019) 048002.

11) N. Mounet, M. Gibertini, P. Schwaller, D. Campi, A. Merkys, A. Marrazzo, T. Sohier, I.E. Castelli, A. Cepellotti, G. Pizzi and N. Marzari: Nat. Nanotechnol. 13 (2018) 246-252.

12) J. Wang, H. Fan and H. Yu: Mater. Trans. 56 (2015) 1911-1914.

13) S. Thomas: Nat. Electron. 1 (2018) 613.

14) H. Kim, H.J. Yun, S. Choi and B.J. Choi: Mater. Trans. 61 (2020) 8893.

15) Z. Al Balushi et al.: Nat. Mater. 15 (2016) 1166-1171.

16) A. Mogulkoc, Y. Mogulkoc, M. Modarresi and B. Alkan: Phys. Chem. Chem. Phys. 20 (2018) 28124.

17) D.C. Camacho-Mojica and F. López-Urías: Sci. Rep. 5 (2016) 17902.

18) D. Wu, M.G. Lagally and F. Liu: Phys. Rev. Lett. 107 (2011) 236101.

19) C.L. Freeman, F. Claeyssens, N.L. Allan and J.H. Harding: Phys. Rev. Lett. 96 (2006) 066102.

20) Q. Peng, C. Liang, W. Ji and S. De: Appl. Phys. A 113 (2013) 483-490.

21) Z. Qin, G. Qin, X. Zuo, Z. Xiong and M. Hu: Nanoscale 9 (2017) 4295-4309.

22) D. Wu, M.G. Lagally and F. Liu: Phys. Rev. Lett. 107 (2011) 236101.

23) G. Kresse and J. Furthmüller: Phys. Rev. B 54 (1996) 11169.

24) P.E. Blöchl: Phys. Rev. B 50 (1994) 17953.

25) G. Kresse and D. Joubert: Phys. Rev. B 59 (1999) 1758.

26) J.P. Perdew, K. Burke and M. Ernzerhof: Phys. Rev. Lett. 77 (1996) 3865.

27) H.J. Monkhorst and J.D. Pack: Phys. Rev. B 13 (1976) 5188

28) S. Grimme, J. Antony, S. Ehrlich and H. Krieg: J. Chem. Phys. 132 (2010) 154104.

29) S. Hong, S.M. Nakhmanson and D.D. Fong: Rep. Prog. Phys. 79 (2016) 076501.

30) M. Xiao, T. Yao, Z.A. Peng Wei, D. Wang and H. Song: Phys. Chem. Chem. Phys. 17 (2015) 8692-8698.

31) P.G. Moses, M. Miao, Q. Yan and C.G. Van de Walle: J. Chem. Phys. 134 (2011) 084703.

32) A. Togo, L. Chaput and I. Tanaka: Phys. Rev. B 91 (2015) 094306.

33) V. Wang, N. Xu, J.C. Liu, G. Tang and W.-T. Geng: VASPKIT: A Preand Post-Processing Program for VASP Code, arXiv:1908.08269.

34) V.N. Tuoc, T.D. Huan and L.T.H. Lien: Phys. Status Solidi B 249 (2012) 1241-1249.

35) G. Makov and M.C. Payne: Phys. Rev. B 51 (1995) 4014.

36) J. Neugebauer and M. Scheffler: Phys. Rev. B 46 (1992) 16067.

37) L. Bengtsson: Phys. Rev. B 59 (1999) 12301.

38) R.F.W. Bader: Atoms in Molecules - A Quantum Theory, (Oxford Univ. Press, Oxford, 1990). 